\title{
Langmuir probe-based observables for plasma-turbulence code validation and application to the TORPEX basic plasma physics experiment
}

\author{
Paolo Ricci, ${ }^{*}$ C. Theiler, A. Fasoli, I. Furno, B. \\ Labit, S.H. Müller ${ }^{1}$, M. Podestà ${ }^{2}$, and F.M. Poli ${ }^{3}$ \\ Centre de Recherches en Physique des Plasmas - École Polytechnique Fédérale de Lausanne, \\ Association EURATOM-Confédération Suisse, CH-1015 Lausanne, Switzerland \\ ${ }^{1}$ Center for Energy Research, University of California, San Diego, CA 92093 \\ 2 Department of Physics and Astronomy, \\ University of California, Irvine, CA 92697 \\ ${ }^{3}$ Center for Fusion, Space and Astrophysics, \\ Department of Physics, University of Warwick, \\ Coventry, CV4 7AL, United Kingdom
}

(Dated: January 12, 2009) 


\begin{abstract}
The methodology for plasma-turbulence code validation is discussed, with focus on the quantities to use for the simulation-experiment comparison, i.e. the validation observables, and application to the TORPEX basic plasma physics experiment [A. Fasoli et al., Phys. Plasmas 13, 055902 (2006)]. The considered validation observables are deduced from Langmuir probe measurements and are ordered into a primacy hierarchy, according to the number of model assumptions and to the combinations of measurements needed to form each of them. The lowest levels of the primacy hierarchy correspond to observables that require the lowest number of model assumptions and measurement combinations, such as the statistical and spectral properties of the ion saturation current time trace, while, at the highest levels, quantities such as particle transport are considered. The comparison of the observables at the lowest levels in the hierarchy is more stringent than at the highest levels. Examples of the use of the proposed observables are applied to a specific TORPEX plasma configuration characterized by interchange-driven turbulence.
\end{abstract}

*Electronic address: paolo.ricci@epfl.ch 


\section{INTRODUCTION}

The validation of plasma turbulence codes plays a fundamental role in the development of magnetically confined fusion, as it is a key step in assessing the maturity of the understanding of the plasma dynamics and the predictive capabilities of simulations. A validation project is a four step procedure [1]. (i) The simulation model needs to be qualified, i.e. it is necessary to establish that for the simulated physical phenomenon, the hypothesis of the model apply. (ii) The verification of the code is necessary, in order to prove that the code solves correctly the model equations. (iii) Simulation and experiment have to be compared considering a number of physical quantities, common to the experimental measurements and simulation results, and analyzed using the same techniques. These physical quantities are denoted as validation observables. (iv) For the identified validation observables, the agreement between simulations and experiments needs to be quantified by using an appropriate metric. The metric should take into account how the errors affecting experimental measurements and simulation results (e.g., errors due to the discretization of the equation for their numerical solution) propagate when the validation observables are formed from experimental measurements and simulation results.

While model qualification and code verification are now routinely considered in plasma physics (see Ref. [2] for some examples) and their methodology has been formulated in considerable detail, only recently the plasma physics community has approached a rigorous methodology for establishing the validation observables and the comparison metric. Herein, we focus on point (iii) of the validation guidelines: the goal of the present paper is to identify and classify the validation observables to use in the simulation-experiment comparison. In particular, we consider measurement provided by Langmuir probes (LPs) and we rank the observables into a primacy hierarchy. The primacy hierarchy tracks how measurements are combined together and models are assumed to obtain a validation observable: observables at a lower level in the hierarchy, such as the statistical and spectral properties of the ion saturation current time traces, combine fewer measurements and require fewer model assumptions; thus, they are more stringent for the comparison [1]. Vice-versa, observables like particle transport that require a higher number of model assumptions are at the highest levels of the primacy hierarchy and are less stringent for the purpose of validation. For the identified observables, the comparison is performed by applying the same data analysis 
techniques to experimental and simulation data.

The identified observables are applied to plasma turbulence code validation in the TORPEX basic plasma physics experiment [3], an ideal configuration for a validation project. TORPEX is a simple magnetized torus (SMT), a relatively simple magnetic configuration in which a vertical magnetic field $B_{z}$ is superposed on a toroidal magnetic field $B_{t}$, creating helical field lines with both ends terminating on the vessel. The plasma is generated and sustained by microwaves in a region that is radially localized and toroidally symmetric. Turbulence driven by curvature and plasma gradients causes plasma transport in the radial direction while, similarly to the scrape-off layer of magnetic fusion devices, plasma is progressively lost in the parallel direction, through losses at the sheath ends. The plasma dynamics can be directly investigated through a wide range of diagnostics covering the whole poloidal cross section. Furthermore, TORPEX flexibility allows wide parameter scans.

The proposed observables for simulation-experiment comparison are used for a specific TORPEX configuration, where the main instability drive is given by the interchange mode, while drift waves play a minor role [4]. Since $k_{\|} \simeq 0$ for the interchange instability, the plasma dynamics can be described by a relatively simple two-dimensional model [6], based on the drift-reduced Braginskii equations [5]. Although the model used is quite crude, its simplicity has been useful to make analytical progress in the study of the properties of the SMT plasma dynamics [6] and it constitutes a good testbed for a practical application of the proposed methodology. The same methodology will be applied to more advanced codes in the future.

The rest of the paper is organized as follows. The observables for the comparison between experimental results and simulations are discussed in Sec. II, where a primacy hierarchy is introduced. In Sec. III, we focus on an example where the identified observables are shown from experimental data and simulations. The concluding remarks follow in Sec. IV. The experimental setup and the model used for the simulation in the example are described in App. A and B, respectively. 


\section{OBSERVABLES FOR THE COMPARISON BETWEEN EXPERIMENTAL AND SIMULATION RESULTS}

When experimental measurements are combined together and models are assumed to provide experimental observables, the errors associated with each measurement or approximations contained in the models can add up or cancel out. Thus, not all the experimentally measured quantities provide equally stringent results to compare with the simulations. For example, experiments and simulations can fortuitously provide similar particle transport, even though the fluctuation levels for $n$ and $\phi$ are inconsistent, because the errors on $n$ and $\phi$ can cancel each other out when their relative phase shift is taken into account to compute transport.

Therefore, it is useful to order the validation observables into a primacy hierarchy that tracks how the measurements are combined together and what assumptions are used in the models employed to form the observables [1]. At the lowest levels in the primacy hierarchy we place the observables that require the smallest number of model assumptions and correspond to the most direct measurements; vice-versa, at the highest levels, we place quantities that require the highest number of assumptions and are obtained by combining different measurements.

We note that one should take into account the model assumptions and the combinations of measurements used to obtain both the experimental and the simulation observables; the primacy hierarchy for experiment-simulation comparison results from the combination of the individual primacy hierarchies for experiment and simulation. Precisely, the resulting primacy level is provided by the sum of the number of assumptions and hypotheses taken to form observables individually from experimental measurements and simulation results. We point out that simulation and experimental primacy hierarchy levels can be different. For example, observables corresponding to direct experimental measurements, like ion saturation current time traces, can often be obtained from simulation results only through the use of synthetic diagnostics and model assumptions that relate quantities like $n, \phi$, and $T_{e}$ to the ion saturation current.

If simulations and experiments are compared using an observable at a certain level in the primacy hierarchy, observables at the lower levels should also be considered, where 


\begin{tabular}{lccc}
\hline \hline & Experimental hierarchy & Simulation hierarchy Comparison hierarchy \\
\hline$I_{\text {sat }}, V_{f l}$ & 1 & 2 & 2 \\
$\bar{T}_{e}, \bar{n}, \bar{\phi}$ & 2 & 1 & 2 \\
$\left(f, k_{z}\right)$ mode density & 2 & 1 & 2 \\
Structure analysis, CAS & 2 & 3 & 4 \\
$T_{e}$ & 3 & 1 & 3 \\
$\Gamma_{\text {struc }}$ & 3 & 4 & 6 \\
$\Gamma$ & 3 & 2 & 4 \\
\hline \hline
\end{tabular}

TABLE I: Primacy hierarchy for the obserables in the TORPEX device.

the comparison is more stringent. The quality of the agreement between simulations and experiments can only be checked by comparing different quantities at different hierarchy levels. To quantify such agreement, an appropriate metric should take into account all the errors associated with the model assumptions and the combinations of observables.

In the following, we discuss the comparison observables and, as summarized in Tab. I, we order them according to the primacy hierarchies. The considered experimental measurements are collected using LPs [7], devices consisting of a conducting refractory material tip drawing the current $I\left(\phi_{b}\right)$ from the plasma, when biased with a voltage $\phi_{b}$. The order of the discussion follows the experimental primacy hierarchy.

\section{A. Experimental primacy level: 1}

By biasing a LP at an increasing negative potential, it is observed that the current drawn from the probe saturates at a value called ion saturation current, $I_{\text {sat }}[7]$. On the other hand, the potential of a floating LP (i.e., a LP drawing no net current from the plasma) is dubbed the floating potential, $V_{f l}$ [7]. Thereby, $I_{s a t}$ and $V_{f l}$ are two observables that can be directly measured by a LP to study the local turbulence properties. They fall in the first level of the primacy hierarchy and they constitute ideal experimental observables for the simulation-experiment comparison.

Simulations do not provide $I_{s a t}$ and $V_{f l}$ directly, since they typically evolve quantities like $n, T_{e}$, and $\phi$; a model for the LP has to be assumed in order to deduce $I_{\text {sat }}$ and $V_{f l}$ from the 
simulation data. Such model has to take into account: (i) how $I_{s a t}$ and $V_{f l}$ depend on the plasma parameters; (ii) the geometrical properties of the LP that influence the measurement of $I_{s a t}$ and $V_{f l}$; (iii) the LP frequency response. Thus, $I_{s a t}$ and $V_{f l}$ are second level observables in the simulation primacy hierarchy.

Once a model for a LP has been applied, simulations and experiments provide time traces of $I_{s a t}$ and $V_{f l}$. For validation purpose, it is then possible to compare quantities like the probability distribution function (PDF) of the time series, its power spectral density (PSD), and the results of more advanced statistical analysis, like the packing fraction [8]. A less refined comparison can focus on the PDF moments, in particular the standard deviation, skewness, and kurtosis (second, third, and forth order moments).

\section{B. Experimental primacy level: 2}

At the second level of the experimental primacy hierarchy, we consider time-averaged quantities, like the density, $\bar{n}$, the potential, $\bar{\phi}$, and the electron temperature $\bar{T}_{e}$, obtained indirectly from LP measurements. We also consider observables concerning global properties of TORPEX turbulence, combining the measurements of multiple LPs.

The time-averaged values $\bar{n}, \bar{\phi}$, and $\bar{T}_{e}$ can be estimated by assuming a model that prescribes a functional dependence of the LP response upon the plasma parameters, i.e. $I\left(\phi_{b}\right)=I\left(\phi_{b} ; \phi, n, T_{e}\right)$. By sweeping $\phi_{b}$, a set of experimental points $\left[\phi_{b}, I\left(\phi_{b}\right)\right]$ is obtained. The best fit of those points, given by the curve $I\left(\phi_{b}\right)=I\left(\phi_{b} ; \bar{\phi}, \bar{n}, \bar{T}_{e}\right)$, provides a way to estimate the time-averaged plasma properties. The use of a $\operatorname{LP}$ model makes $\bar{\phi}, \bar{n}$, and $\bar{T}_{e}$ second level observables in the experimental primacy hierarchy.

While observables related to global properties of the plasma dynamics can be directly obtained from the simulation data (e.g., through the Fourier analysis of simulation results), in the experiment the limited number of probes that can be inserted in the plasma makes global properties of turbulence a less direct quantity to observe. A number of techniques are currently employed in the TORPEX device for the study of global properties.

The two point correlation technique [9] is used to identify the wavelength and the frequency of the modes present in the experiment [10]. For this purpose, the signals from two LPs operated in $I_{\text {sat }}$ mode are considered and the phase shift between them is evaluated. If 
stationary and homogeneous turbulence is considered, characterized by a weak nonlinearity and a bi-univocal relation between the frequency of the mode and its wavelength, from the phase shift between the two probes it is possible to estimate a histogram of the fluctuations in the $(k, f)$ space, $k$ and $f$ being the wavevector in a specified direction and the frequency of a mode. We remark that the two point correlation technique is a local measurement that, under the assumption of homogeneity, provides global turbulence observables. Since the technique is based on combining the $I_{\text {sat }}$ measurements from two LPs, the mode occupancy of the $(k, f)$ space is an observable that holds the second level in primacy hierarchy. If the two point correlation technique is applied to the simulation data, by considering two virtual LPs in $I_{\text {sat }}$ mode, the results hold the third level in the primacy hierarchy. However, the mode occupancy of the $(k, f)$ plane is a first level observable, if it is evaluated based on the space and time Fourier analysis of quantities like $n, \phi$, or $T_{e}$.

The conditional average (CA) technique [11] is particularly suited to study the twodimensional spatio-temporal dynamics of blobs, structures of increased plasma density, that move from the source region of TORPEX, where the plasma density is higher, to the low field side of the machine, characterized by lower plasma density [12]. Similarly to the plasma edge of all laboratory devices, blobs give TORPEX transport an intermittent character. In order to evaluate the conditionally averaged blob, a LP located on the TORPEX low field side and operated in $I_{s a t}$ mode is taken as a reference signal, and a set of trigger times $t_{i}$ is found, corresponding to $I_{\text {sat }}$ exceeding a threshold value (supposedly, when a blob passes by the probe). We evaluate the CA of the fluctuating part of the $I_{\text {sat }}$ signal, i.e. $\tilde{I}_{\text {sat }}=I_{\text {sat }}-\bar{I}_{\text {sat }}$, as

$$
\left\langle\tilde{I}_{s a t}\right\rangle_{c a}(r, z, \tau)=\frac{1}{N} \sum_{i=1}^{N} \tilde{I}_{s a t}\left(r, z, t_{i}+\tau\right)
$$

where $N$ is the number of detection events. We denote with $r$ the radial coordinate, while $z$ is the vertical direction $(r=0$ and $z=0$ at the torus axis). The CA technique is usually less convenient for simulation than for experimental data, since finding a sufficient number of trigger events requires long time series that are computationally expensive. As the CA combines multiple first level observables, it occupies the second level in the primacy hierarchy. On the other hand, $\tilde{I}_{s a t} \mathrm{CA}$ occupies the third level in the simulation primacy hierarchy, since the $I_{\text {sat }}$ signals have to be obtained using a LP model.

In the analysis of TORPEX data, pattern recognition techniques combined with statistical 
analysis are applied to study the dynamics of positive and negative structures [13], i.e.

regions where the $\tilde{I}_{\text {sat }}$ signal is bigger or smaller than the threshold value $I_{\text {sat,tr }}\left(\tilde{I}_{\text {sat }}>I_{\text {sat }, t r}\right.$ for positive structures and $\tilde{I}_{s a t}<-I_{\text {sat,tr }}$ for negative structures). The technique, the details of which are described in Ref. [14], can identify the presence of structures, their trajectory, dimension, amplitude, and velocity. It is then possible to use the information gathered for a statistical treatment. We note that a limit of the structure analysis is imposed by the LP array grid spacing, which cannot be reduced over a certain limit for technical reasons. This implies, for example, that structures whose size is below a certain value cannot be detected. On the other side, simulations offer the opportunity to refine the LP grid as desired, and study the structure analysis convergence with the grid refinement. Structure analysis is a second and third level observable in the experimental and simulation primacy hierarchies, respectively.

\section{Experimental primacy level: 3}

Measurement of $T_{e}$ fluctuations and transport are included in the third level of the primacy hierarchy. On TORPEX, these involve the use of a three-tip probe array (TP) [15], a set of three closely spaced LPs that are operated in different regimes. TP measurements are delicate. They are complicated by probe shadowing, phase delay, and decorrelation effects. Different ways to circumvent these issues have been proposed (see [16] and references therein) that we do not consider for the purpose of the present work.

For $T_{e}$ measurements, the $\mathrm{TP}$ is operated as a triple proble, i.e. one tip is operated in $V_{f l}$ mode. The other two, floating with respect to the ground, are connected to each other through a fixed voltage source (the tip voltages are indicated with $V^{+}$and $V^{-}, \Delta V=$ $\left.V^{+}-V^{-}\right)$. If one assumes similar plasma properties for the three tips, and that the $V^{+}$ tip current can be modeled as $I^{+}=n f\left(T_{e}, V_{f l}, V^{+}\right)$[and similarly $I^{-}=n f\left(T_{e}, V_{f l}, V^{-}\right)$, by noticing that $I^{+}+I^{-}=0$, one obtains the equation $f\left(T_{e}, V_{f l}, V^{+}\right)+f\left(T_{e}, V_{f l}, V^{-}\right)=0$ that provides the $T_{e}$ time trace, since $V_{f l}, V^{+}$, and $V^{-}$are measured quantities. $T_{e}$ is thus a third level observable in the experiment while $T_{e}$ time trace is a a first level observable in the simulation primacy hierarchy.

A number of advanced techniques are used in TORPEX in order to evaluate particle 
transport [17]. For the purpose of the present paper, we consider a simplified measurement for the flux, where two tips of the $\mathrm{TP}$ are operated in the $V_{f l}$ regime (the vertical distance between them is $d$ ) and the third one (located between the other two) is in $I_{\text {sat }}$ mode. Time-averaged transport is evaluated as

$$
\Gamma=\frac{1}{B} \overline{I_{s a t} \frac{\partial \phi}{\partial z}} \simeq \frac{1}{B} \overline{I_{s a t} \frac{\partial V_{f l}}{\partial z}} \simeq \frac{1}{B} \overline{I_{s a t} \frac{\Delta V_{f l}}{d}}
$$

where $\Delta V_{f l}$ is the difference between the two $V_{f l}$ measurements. Since the above technique assumes that $\overline{I_{s a t} \partial\left(V_{f l}-\phi\right) / \partial z} \simeq 0$, and measurements from multiple LPs are employed, $\Gamma$ is a third level observable in the primacy hierarchy. It belongs instead to the second level for what concerns the simulation results.

Transport properties in the TORPEX device can also be investigated through the structure analysis results $[13,14]$. In order to evaluate the time-averaged structure-induced transport, $\Gamma^{s t r}$, one evaluates the plasma mass carried by all the structures crossing a virtual surface over an observational time. The particle flux is then obtained by dividing the total mass by the surface area and the observational time. Since $\Gamma^{s t r}$ combines a number of measurements that are the result of the structure analysis, it occupies the third level in primacy hierarchy. The analysis technique leading to $\Gamma^{s t r}$ can also be applied to simulation data; in this case, this observable is at the forth level in the primacy hierarchy.

\section{EXAMPLES OF SIMULATION AND EXPERIMENT COMPARISON}

We present some examples of comparison between experimental data and simulation results, performed by using the observables discussed in the previous section. We consider a single plasma configuration; the experimental setup and the diagnostics used (HEXTIP, SLP, and the TP) are described in App. A, while the simulation model is discussed in App. B. We remark that this configuration is characterized by turbulence with an interchange character, i.e. $k_{\|} \simeq 0$ [4], thus the plasma dynamics can be studied by a relatively simple two-dimensional model [6]. The presence of the vessel is taken into account through sheath losses in the parallel direction, while its effects on the perpendicular dynamics are neglected. We focus our comparison effort on the TORPEX midplane $(z=0)$, where vessel effects on the perpendicular dynamics are minimized. 
To model the LP response we use Bohm's model [18],

$$
I\left(\phi_{b}\right)=\frac{1}{2} e n c_{s} A\left[1-\exp \left(\Lambda+e \frac{\phi_{b}-\phi}{T_{e}}\right)\right]
$$

where $A$ is the area of the LP (in the present case, $A=1.3 \cdot 10^{-5} \mathrm{~m}^{2}$ for the HEXTIP

probes, $A=1.8 \cdot 10^{-5} \mathrm{~m}^{2}$ for the SLP probes, and $A=2 \cdot 10^{-5} \mathrm{~m}^{2}$ for the TP tips), and the value $\Lambda=\log \sqrt{m_{i} /\left(2 \pi m_{e}\right)}$ is deduced from the theory [18]. We note that $\Lambda$ can also be derived from experimental data to account for the actual probe geometry [19]. For the simulation, we have performed numerical tests to take into account the real LP dimensions, by averaging the $I_{\text {sat }}$ signal over the finite LP surface. We have observed that taking into account finite LP dimension leads to a damping of fluctuation frequencies greater than 20 $\mathrm{kHz}$ (corresponding to short wavelength modes). The high frequency modes filtered out have a small impact on the plasma dynamics; thus, in the rest of the paper, we consider a LP point-like model to interpret simulation results. Following the experiment, an acquisition frequency of $250 \mathrm{kHz}$ is used in the simulation, and the simulated LP time trace is low-pass filtered with a cutoff frequency at $30 \mathrm{kHz}$, mimicking the bandwidth frequency response of the electronics associated with the LPs.

\section{A. Experimental primacy level: 1}

\section{1. $I_{\text {sat }}$ Measurements}

Figures 1 and 2 illustrate $I_{\text {sat }}$ measurements, comparing HEXTIP and simulation values (in the latter case, the model in Eq. (3) is used, i.e. $I_{s a t}=e n c_{s} A / 2$ ). In Fig. 1, the PDF and the PSD of $I_{\text {sat }}$ are displayed at different locations. Near the source region, i.e. at $r=-4 \mathrm{~cm}$, both experimental and simulated PDFs are slightly negatively skewed, while they become positively skewed on the low field side of the machine, indicating the presence of intermittent transport events. While the PSD shows that $I_{s a t}$ is dominated at $r=4 \mathrm{~cm}$ by a coherent mode with a frequency of about $8 \mathrm{kHz}$ in TORPEX, the presence of a single coherent mode is less clear in the simulation, and only a weak signature of its presence is apparent at $r=12 \mathrm{~cm}$.

The moments of the experimental and simulated PDF of $I_{\text {sat }}$ are compared in Fig. 2. The average values of $I_{\text {sat }}$ are peaked at the UH resonance layer [20], where most of the 
plasma is produced, with the simulation peak value about $50 \%$ smaller than the experimental one. It is also shown that $I_{\text {sat }}$ decays on the TORPEX low field side, with a scale length about a factor of two longer in the simulation than in the experiment. The simulation fluctuation level is about a factor of two lower than the experimental one, and about constant across the SMT cross section. The normalized third and forth moments, skewness and kurtosis, of the $I_{\text {sat }}$ time trace show similar trends: the profiles tend to be more skewed and show a larger deviation from a gaussian shape on the low field side, moving away from the source region. Although deviations from gaussian values occur on a shorter spatial scale in the experiment than in the simulation, similar universality properties are displayed. This is shown in the inset of Fig. 2, where, as previously observed in the experimental data, a parabolic dependence between skewness $(S)$ and kurtosis $(K)$ is found, expressed as $K \simeq 2.78+1.5 S^{2}[21]$

\section{2. $V_{f l}$ measurements}

Bohm's model states that $V_{f l}=\phi-\Lambda T_{e} / e$. By using this expression, we have compared the experimental and simulation values of $V_{f l}$ in Fig. 3, focusing on the time-average, standard deviation, skewness, and kurtosis. In order to preserve quasi-neutrality, an equal number of ions and electrons leave from the SMT, and Bohm's sheath conditions show that this occurs when $\phi-\Lambda T_{e} / e=V_{f l} \simeq 0$; thus, we expect $V_{f l}$ to be a relatively small quantity in comparison to $\phi$. The value of $V_{f l}$ provided by both the simulation and the experiment is about $10 \%$ of $\phi$. We should note that, in the simulation data, cancellation errors strongly affect the $V_{f l}$ evaluation since $V_{f l}$ is obtained as the difference between $\Lambda T_{e}$ and $\phi$, two quantities that are in fact very close to each other. On the experimental side, $V_{f l}$ measurements are strongly influenced by fast electrons [18], whose presence is not taken into account by the simulation. Because of the difficulties involving both experimental and simulation $V_{f l}$ measurements, we conclude that, at least in an open field line configuration like the SMT, $V_{f l}$ has a limited use as a validation observable. 


\section{B. Experimental primacy level: 2}

1. $\bar{n}, \bar{\phi}$, and $\bar{T}_{e}$

In general, $\bar{n}, \bar{\phi}$, and $\bar{T}_{e}$ are evaluated by sweeping the $\phi_{b}$ potential. In TORPEX, the $\phi_{b}$ sweeping is from $-30 \mathrm{~V}$ to $10 \mathrm{~V}$, with a frequency of $330 \mathrm{~Hz}$ (we note that the typical time scale of turbulence, i.e. $c_{s} / \sqrt{R L_{p}} \sim 100 \mathrm{kHz}$ occurs on a much faster time scale than the sweeping cycle). In Fig. 4, we show the typical $\left[\phi_{b}, I\left(\phi_{b}\right)\right]$ points obtained through a LP voltage sweeping and the $I\left(\phi_{b}\right)=I\left(\phi_{b} ; \bar{\phi}, \bar{n}, \bar{T}_{e}\right)$ curve from Bohm's model that best fits the experimental data.

The time average of the simulated $\bar{n}, \bar{\phi}$, and $\bar{T}_{e}$ profiles is shown in Fig. 5. Simulation and experimental peak values show good agreement. On the low field side of the device, the agreement deteriorates, since the simulation scale lengths are about twice as long as the experimental ones. Besides the time-average, also for simulation data it is possible to follow the experimental technique to evaluate $\bar{n}, \bar{\phi}$, and $\bar{T}_{e}$; this can be done in order to verify the routines used for the experimental data analysis. For this purpose, a synthetic LP is inserted in the simulation domain and its response is best-fitted by using the same algorithms that are routinely employed to fit experimental $\left[\phi_{b}, I\left(\phi_{b}\right)\right]$ curves. In Fig. 4 , the $\left[\phi_{b}, I\left(\phi_{b}\right)\right]$ points from the synthetic probe, and the best fitting curve of Eq. (3) are shown. Figure 5 shows $\bar{n}, \bar{\phi}$ and $\bar{T}_{e}$ evaluated from the simulation following this experimental technique (five virtual sweeping cycles are used). The successful verification observed by comparing the parameters directly obtained from the simulation and those reconstructed through the synthetic LP gives us confidence on the data analysis techniques typically used to study experimentally the TORPEX plasma.

\section{Two point correlation technique}

The results of the two point correlation technique, applied both to simulation and experimental results, are shown in Fig. 6. Clear results are observed in the experimental data: the dominant mode has $k_{z} \simeq k_{\Delta}\left(k_{\Delta}=2 \pi / \Delta\right.$, with $\Delta=2 \pi R B_{v} / B_{\phi}$ the return distance of a field line in the poloidal plane), in agreement with the property of the interchange mode of growing on the longest allowed spatial scales [4]. The frequency of the mode is about 8 
$\mathrm{kHz}$, corresponding to the value of the $\mathbf{E} \times \mathbf{B}$ frequency, i.e. $\mathbf{v}_{\mathbf{E} \times \mathbf{B}, z} / \Delta$.

The simulation results are less clear than the experimental ones and the application of more advanced spectral techniques, like the wavelet analysis, provides similar results. This is due to the broader turbulence spectrum in the simulation, indicating stronger nonlinear interactions between the modes. We note that the turbulence spectrum can also be obtained from direct Fourier analysis of the simulation results, since the plasma properties are known in the whole simulation domain. The Fourier analysis (not shown here) confirms that, similarly to the experimental results, the dominant mode of the system has $k_{z}=k_{\Delta}$ with frequency of about $6 \mathrm{kHz}$, corresponding to the $\mathbf{E} \times \mathbf{B}$ frequency.

\section{CA for blob analysis}

The CA analysis is performed by considering a LP reference probe located at $r=10 \mathrm{~cm}$ and $z=0$, and a threshold value of $3 \sigma, \sigma$ being the standard deviation of the reference signal. In the time window considered in the simulation $(\simeq 0.1 \mathrm{~s})$, about 20 events are identified. This number is about the double than for the experimental data, which is consistent with the lower skewness observed in the simulation results. We note that this is a relatively small number of trigger events to obtain good statistical convergence and to perform a deep experiment-simulation comparison. The results of the CA is shown in Fig. 7.

While their rise times are different, the averaged reference signals show similar decay times in the simulation and in the experiment. The two-dimensional blob dynamics displays a much more coherent signal in the experiment than in the simulation, in agreement with the findings of the two point correlation technique.

\section{Structure analysis}

For the structure analysis, the threshold value $I_{s a t, t r}$ is chosen as the average of the standard deviations of all the HEXTIP probe signals [14]. Only structures that last longer than 30 time frames (i.e., $120 \mu \mathrm{s}$ ) are considered in a time window of about $0.1 \mathrm{~s}$. In Fig. 8, we plot a number of quantities that are the results of the structure analysis. In particular, the number of structures, $N$, the vector representing their averaged velocity, $\mathbf{V}$, the average amplitude, $H$, and the average area, $A$, are shown for both positive and negative structures 
(denoted with the '+' and '-' superscripts, respectively).

We find that $N$ is about five times smaller in the simulation data than in the experiment. V shows an outward radial motion for positive structure, while negative structures move inwards. $H$ is twice as large in the experiment than in the simulation, while $A$ is about the same. The simulation allows a convergence study of the structure analysis with the grid size. By refining the grid spacing between the synthetic probes by a factor of two in both directions, we find that while $N$ doubles, $\mathbf{V}, H$, and $A$ remain about the same. This shows that the smaller structures detected only by the higher resolution grid have similar statistical properties as the larger ones.

\section{Experimental primacy level: 3}

1. $T_{e}$

Bohm's model indicates that $I\left(\phi_{b}\right)$ is proportional to $n$; thus, $T_{e}$ measurements can be obtained from a TP. If the applied potential $\Delta V \gg T_{e} / e$, and $I_{\text {sat }}$ saturates for a sufficiently negative potential, from Bohm's model one has $T_{e} \simeq e\left(V^{+}-V_{f l}\right) / \log (2)$. In Fig. 9, we compare the profiles of the $T_{e}$ standard deviations. Experiment and simulation provide comparable levels of $T_{e}$ fluctuations although the details of the profiles are somewhat different: in particular the experimental $T_{e}$ fluctuations are bigger than the simulated in the source region, while they become smaller on the low field side.

\section{2. $\Gamma^{s t r}$}

The structure-induced transport is shown in Fig. 10. For the structure analysis, LPs are operated in $I_{\text {sat }}$ mode and, to make the comparison with measurements from other LPs easier, we consider $J_{\text {sat }}=I_{\text {sat }} / A$ as the transported quantity ( $A$ is the LP surface area). Since both $N$ and $H$ are smaller in the simulation than in the experiment, the structure-induced transport in the simulation is sensibly smaller. 
3. $\Gamma$

The $J_{\text {sat }}$ transport measurement provided by the TP is shown in Fig. 10, and compared with the simulated value. Both the experimental and the simulated transport have been evaluated according to Eq. (2) (numerical tests show that using $V_{f l}$ to evaluate the particle transport instead of $\phi$ leads to an overestimate of the flux by 50\%). Simulation and experiment show similar peak values of the transport but different scale lengths. One also notices that $\Gamma \gg \Gamma^{s t r}$; i.e., most of the plasma transport in this plasma scenario is not associated to the motion of coherent plasma structures, as defined in the parameters of this work.

\section{DISCUSSION AND CONCLUSIONS}

The methodology for plasma turbulence code validation has been discussed, with focus on the observables for experiment-simulation comparison. We have considered the measurements provided by LPs in the basic plasma physics experiment TORPEX.

We have built a primacy hierarchy that considers how measurements are combined together and models are assumed. The agreement between simulation results and experimental data is characterized by comparing the observables at the different levels of the primacy hierarchy, obtained by employing the same data analysis techniques. The lowest level of the experimental primacy hierarchy, corresponding to direct measurements of time traces of $V_{f l}$ and $I_{\text {sat }}$, involves no model assumptions nor combinations of measurements, thus naturally provides observables for the comparison. However, simulations do not provide $I_{s a t}$ or $V_{f l}$ directly, but a model is needed to extract them from typical simulation output $\left(n, T_{e}\right.$, and $\left.\phi\right)$. Moreover, the physical meaning of these observables is not immediate. At the highest level of the hierarchy considered in the present paper, there are observables like particle transport and $T_{e}$ fluctuations. These can be obtained by assuming a model for the LP response, and

combining measurements from more than one probe. Higher level observables have a more immediate physical meaning and are directly obtained from simulation results.

We have discussed an example of comparison of experimental results and simulation output that makes use of the proposed observables, considering a specific TORPEX configuration where the plasma is characterized by interchange-driven turbulence, in principle well described by a two-dimensional simulation code. Despite the crudeness of the simu- 
lation model, the order of magnitude of all the observables is the same in simulation and experimental data. A detailed comparison of the lowest level observables (i.e., $I_{s a t}$ and $V_{f l}$ time traces) shows that experiments are dominated in the source region by a strong coherent mode, whose signature is considerably weaker in the simulation. The comparison also shows that the experimental scale length of $I_{\text {sat }}$ is larger in the simulations than in the experiment by a factor of about two, and that the intermittent events occurring on the low field side of the machine play a more significant role on plasma transport in the experiment than in the simulation. The observables at the second level of the hierarchy reveal smaller experimental $\bar{n}$ and $\bar{T}_{e}$ scale lengths. The reliability of this observation is strengthened by the previous comparison of the $I_{\text {sat }}$ scale lengths. The two point correlation technique confirms and quantifies the difference in the mode coherence observed between the simulation and the experiment. The broader frequency spectrum of the turbulence in the simulation leads to fewer long-lived and high amplitude structures, and smaller $\Gamma^{s t r}$. Instead, the simulation and the experiment show comparable values of $\Gamma$.

The comparisons between simulation and experiment across the primacy hierarchy suggests that the main disagreements between simulation and experiment are due to a different coherency of the $k_{z}=k_{\Delta}$, mode. This causes, for example, a turbulent dynamics with a broader frequency spectrum and a smaller number of coherent structures in the simulation. The different coherency level is possibly associated with the presence of short wavelength and high frequency modes that seem damped in the experiment, while they disrupt large scale structures in the simulation. Possible physical effects missing in the simulation that could explain the different dynamics include three-dimensional modes, the impact of the vessel on the perpendicular dynamics, and the presence of neutrals. On this respect, we expect three-dimensional modes, i.e. drift waves, to develop on spatial scales of the order of $\rho_{s}$, smaller than the typical large global spatial scales of the interchange mode. Another possible origin of experiment-simulation discrepancy resides in the plasma source at the upper hybrid (UH) resonance. The location of the UH resonance depends non linearly on the instantaneous plasma density profile. This could give rise to a positive feedback of the density fluctuations, increasing the coherency of the $k_{z}=k_{\Delta}$ mode that has the highest amplitude. On the other hand, the difference in the equilibrium $n$ and $T_{e}$ spatial scales of the simulation and experiment are possibly due to the simplified simulation geometry, which 
does not take into account the shortening of the connection length with $r$, on the low field side of the machine. The impact of the missing physical effects can be explored by more advanced simulations. Conversely, the experimental conditions could be made closer to the simulation model by reducing the level of neutrals in TORPEX.

The work described in the present paper constitutes a first step in establishing the validation methodology for the TORPEX experiment. The proposed methodology will be completed by the definition of a metric to quantify the agreement between simulation and experimental results, which will take into account the errors associated with model assumptions and the combination of measurements, i.e. the level of each observable in the comparison hierarchy. The methodology will be applied to validate the results of more advanced three-dimensional codes that are currently under development. We expect that this procedure will lead us to a better physical understanding and modeling of plasma turbulence in the TORPEX device and, ultimately, in more complicated magnetic confinement configurations.

\section{ACKNOWLEDGMENTS}

This work is partly supported by the Fonds National Suisse de la Recherche Scientifique. P.R. is supported by a EURATOM fusion fellowship; F.M.P. acknowledges financial support from a United Kingdom Royal Society International Short Visit Grant.

\section{Appendix A: The experimental setup}

The experiments are performed in TORPEX, a toroidal device with major radius $R=1$ $\mathrm{m}$, minor radius $a=0.2 \mathrm{~m}$. A hydrogen plasma is produced and sustained by microwaves in the electron cyclotron range of frequencies (a microwave power of $400 \mathrm{~W}$ is used). Using the technique discussed in Ref. [20], it is observed that the plasma production is localized at the EC and UH layers, that are vertically elongated around $r=-13 \mathrm{~cm}$ and $r=-2 \mathrm{~cm}$, respectively.

A toroidal magnetic field $B_{\phi}=76 \mathrm{mT}$ on axis is used, with a vertical magnetic field $B_{z}=1.7 \mathrm{mT}$. This results in $\Delta=14 \mathrm{~cm}$, where $\Delta=2 \pi R B_{z} / B_{t}$ is the vertical return distance of a field line in the poloidal plane. The configuration results in a vertically elongated 
plasma, where waves are shown to have an interchange character with $k_{\|} \simeq 0$. Typical plasma parameters are in the range $n \simeq 10^{16} \mathrm{~m}^{-3}, T_{e} \simeq 5 \mathrm{eV}, T_{i} \ll T_{e}$.

Diagnostics of the plasma dynamics used here include: (i) the HEXTIP array, a twodimensional hexagonal LP array covering the whole poloidal cross section, with spatial resolution of $3.5 \mathrm{~cm}$ [22]; (ii) the SLP array, a linear array of $8 \mathrm{LPs}$, with $1.8 \mathrm{~cm}$ distance between tips; (iii) a triple probe, with distance between tips of $3.5 \mathrm{~mm}$. The acquisition frequency is $250 \mathrm{kHz}$.

\section{Appendix B: The simulation model}

Owing to the low TORPEX plasma temperature and neglecting collision with neutrals, the drift-reduced Braginskii equations (see, e.g. [5]) can be used to model the plasma dynamics. Since $k_{\|} \simeq 0$, simple two-dimensional fluid equations that describe the plasma turbulence can be used. We assume $B_{z} \ll B_{t}$ so that $B \simeq B_{0} R /(R+r)$, and note that magnetic curvature is constant along a field line and equal to $R+r$. The Braginskii equations are integrated in the parallel direction in order to evolve the line-integrated density, $n(r, z)=$ $\int \mathcal{N}\left(r, z, x_{\|}\right) d x_{\|} / L_{c}$, potential, $\phi(r, z)=\int \Phi\left(r, z, x_{\|}\right) d x_{\|} / L_{c}$, and temperature, $T_{e}(r, z)=$ $\int \mathcal{T}_{e}\left(r, z, x_{\|}\right) d x_{\|} / L_{c}, L_{c}=2 \pi N R$ being the magnetic field line length. We use Bohm's boundary conditions to take into account the ion and electron parallel flow at the sheath edge: by assuming that the density at the edge is equal to $n(r, z) / 2$, it is possible to approximate the ion and electron flows as $\Gamma_{\|, i}=n c_{s} / 2$ and $\Gamma_{\|, e}=n c_{s} \exp \left(-e \phi / T_{e}+\Lambda\right) / 2$, with $\Lambda=$ $\log \sqrt{m_{i} /\left(2 \pi m_{e}\right)}$ [18]. Furthermore, we make use of the Boussinesq approximation [23] for the polarization drift, i.e.,

$$
\nabla \cdot\left(\frac{n m_{i}}{e B} \frac{d}{d t} \frac{\nabla \phi}{B}\right)=\frac{n m_{i}}{e B^{2}} \frac{d}{d t} \nabla^{2} \phi
$$

The evolution equations for $n, \phi$, and $T_{e}$ thus become

$$
\begin{array}{r}
\frac{\partial n}{\partial t}=\left[\frac{c \phi}{B_{0}}, n\right]+\frac{2 c}{e R B_{0}}\left(n \frac{\partial T_{e}}{\partial z}+T_{e} \frac{\partial n}{\partial z}-e n \frac{\partial \phi}{\partial z}\right) \\
+D \nabla^{2} n-\frac{\sigma n c_{s}}{R} \exp \left(\Lambda-e \phi / T_{e}\right)+S_{n}, \\
\frac{\partial \nabla^{2} \phi}{\partial t}=\left[\frac{c \phi}{B_{0}}, \nabla^{2} \phi\right]+\frac{2 B_{0}}{c m_{i} R}\left(\frac{T_{e}}{n} \frac{\partial n}{\partial z}+\frac{\partial T_{e}}{\partial z}\right) \\
+\nu \nabla^{4} \phi+\frac{\sigma c_{s} m_{i} \Omega_{i}^{2}}{e R}\left[1-\exp \left(\Lambda-e \phi / T_{e}\right)\right],
\end{array}
$$




$$
\begin{aligned}
& \frac{\partial T_{e}}{\partial t}=\left[\frac{c \phi}{B_{0}}, T_{e}\right]+\frac{4 c}{3 e R B_{0}}\left(\frac{7}{2} T_{e} \frac{\partial T_{e}}{\partial z}+\frac{T_{e}^{2}}{n} \frac{\partial n}{\partial z}-e T_{e} \frac{\partial \phi}{\partial z}\right) \\
& +k_{e} \nabla^{2} T_{e}-\frac{2}{3} \frac{\sigma T_{e} c_{s}}{R}\left[1.71 \exp \left(\Lambda-e \phi / T_{e}\right)-0.71\right]+S_{T}
\end{aligned}
$$

where $S_{n}$ and $S_{T}$ represent particle and heat sources, $\sigma=R / L_{c}=\Delta /\left(2 \pi L_{v}\right)$, and $[a, b]=$ $\partial_{x} a \partial_{z} b-\partial_{z} a \partial_{x} b$. We note that a similar system of equations has been used in Ref. [24]. The boundary conditions are periodic along the vertical direction (due to the flute property of the interchange mode) and we use Dirichelet boundary conditions in the radial direction.

For the simulation described in the present paper, the experimental values $R=1 \mathrm{~m}$, $\Delta=14 \mathrm{~cm}, \sigma=0.056, \Lambda=3$ are used. The value of the diffusion coefficients are $D=k_{e}=0.064 \mathrm{~m}^{2} / \mathrm{s}$ and $\nu=0.03 \mathrm{~m}^{2} / \mathrm{s}$ (estimates of the realistic values of the diffusion coefficient provide $D=0.001 \mathrm{~m}^{2} / \mathrm{s}$ and $\nu=0.058 \mathrm{~m}^{2} / \mathrm{s}$ ). We have used source profiles that mimic the electron cyclotron (EC) and UH resonance layer in TORPEX, i.e., $S_{n, T}=S_{0 ; n, T}\left\{S_{U H} \exp \left[-\left(r-r_{U H}\right)^{2} / \lambda_{U H}^{2}\right]+S_{E C} \exp \left[-\left(r-r_{E C}\right)^{2} / \lambda_{E C}^{2}\right]\right\}$, with $S_{U H}=1.5$, $S_{E C}=1, \lambda_{U H}=1 \mathrm{~cm}, \lambda_{E C}=0.5 \mathrm{~cm}, r_{U H}=-2 \mathrm{~cm}, r_{E C}=-6 \mathrm{~cm}$, and values of the source strength $\left(S_{0 n}=1.5 \cdot 10^{20} \mathrm{~m}^{-3} \mathrm{~s}^{-1}, S_{0 T}=3.5 \cdot 10^{4} \mathrm{eV} / \mathrm{s}\right)$ estimated experimentally through a global balance of the TORPEX plasma. We remark that dependence of the UH resonance position on $n$ is neglected in the present model.

The system of equations has been solved numerically, using a numerical code developed from the ESEL code [25]. The algorithm used is described in Ref. [26]. The simulation is started from random noise. Then, the sources introduce plasma and heat, increasing the plasma pressure and triggering the interchange instability. The interchange instability leads to density and particle transport in the radial direction from the source region to the low field side of the machine; at the same time, plasma is removed from the system by parallel losses. The results discussed in the present paper focus on the quasi-steady state period, established after the initial simulation transient, as a result of a balance between parallel losses, perpendicular transport, and sources.

The plasma dynamics described by the model (B2-B4) has been discussed in Ref. [6], where the presence of two turbulence regimes was pointed out. The high confinement regime ( $\mathrm{H}$ mode) is characterized by a strong shear flow that creates a transport barrier and can limit the perpendicular transport, while in the low confinement regime (L mode), shear flow 
plays a negligible role. The simulation discussed here falls in the L mode regime.

[1] P.W. Terry, M. Greenwald, J.-N. Leboeuf, G.R. McKee, D.R. Mikkelsen, W.M. Nevins, D.E. Newman, D.P. Stotler, Task Group on Verification and Validation, U.S. Burning Plasma Organization, and U.S. Transport Task Force, Phys. Plasmas 15, 062503 (2008).

[2] A.M. Dimits, G. Bateman, M.A. Beer, B. I. Cohen, W. Dorland, G. W. Hammett, C. Kim, J. E. Kinsey, M. Kotschenreuther, A. H. Kritz, L. L. Lao, J. Mandrekas, W. M. Nevins, S. E. Parker, A. J. Redd, D. E. Shumaker, R. Sydora, and J. Weiland, Phys. Plasmas 3969 (2000); J. Birn, J.F. Drake, M.A. Shay, B.N. Rogers, R.E. Denton, M. Hesse, M. Kuznetsova, Z.W. Ma, A. Bhattacharjee, A. Otto, and P.L. Pritchett, J. Geophys. Res. 106, 3715 (2001); P. Ricci, J.U. Brackbill, W. Daughton, and G. Lapenta, Phys. Plasmas 8, 4102 (2004); V. Umansky, M.S. Day, and T.D. Rognlien, Num. Hear Transfer, Part B 47, 533 (2005); J.R. Myra, D.A. D'Ippolito, D.A. Russel, and M. Umansky, Bull. Am. Phys. Soc. 52, 533 (2007).

[3] A. Fasoli, B. Labit, M. McGrath, S. H. Müller, G. Plyushchev, M. Podestà, and F. M. Poli, Phys. Plasmas 13, 055902 (2006).

[4] F.M. Poli, P.Ricci, A. Fasoli, and M. Podestà, Phys. Plasmas 14052311 (2007).

[5] A. Zeiler, J.F. Drake, and B. Rogers, Phys. Plasmas 4, 2134 (1997).

[6] P. Ricci, B.N Rogers, and S. Brunner, Phys. Rev. Lett. 100, 225002 (2008).

[7] I. H. Hutchinson. Principles of plasma diagnostics. Cambridge University Press, New York, 1987; F. F. Chen. Plasma diagnostic techniques. R. H. Huddlestone and S. L. Leonard, Academic Press, New York, 1965.

[8] M. Onorato, R. Camussi, and G. Iuso, Phys. Rev. E 61, 1447 (2000); M. Agostini, S. J. Zweben, R. Caravazzana, P. Scarin, and G. Serianni, Phys, Plasmas 14, 102305 (2007).

[9] J. M. Beall, Y. C. Kim, and E. J. Powers. J. Appl. Phys. 53, 3933 (1982); N. Iwama, and T. Tsukishima, Appl. Phys. Lett. 31, 783 (1977); S.J. Levinson, J.M. Beall, E.J. Powers, and R.D. Bengtson. Nucl. Fusion 24, 527 (1984); N. Iwama, Y. Ohba, and T. Tsukishima, J. Appl. Phys. 50, 3197 (1979).

[10] F. M. Poli, S. Brunner, A. Diallo, A. Fasoli, I. Furno, B. Labit, S.H. Müller, G. Plyushchev, M. Podestà, Phys. Plasmas 13, 102104, 2006. 
[11] H. Johnsen, H. L. Pecseli, and J. Trulsen, Phys. Fluids 30, 2239 (1987).

[12] C. Theiler, A. Diallo, A. Fasoli, I. Furno, B. Labit, M. Podestà, F. M. Poli, and P. Ricci, Phys. Plasmas 15, 042303 (2008); I. Furno, B. Labit, M. Podestà, A. Fasoli, S. H. Müller, F. M. Poli, P. Ricci, C. Theiler, S. Brunner, A. Diallo, and J. Graves, Phys, Rev. Lett. 100, 055004 (2008); I. Furno, B. Labit, A. Fasoli, F. M. Poli, P. Ricci, C. Theiler, S. Brunner, A. Diallo, J.P. Graves, M. Podestà, and S.H. Müller, Phys. Plasmas 15, 055903 (2008).

[13] S.H. Müller, A. Diallo, A. Fasoli, I. Furno, B. Labit, and M. Podestà, Phys. Plasmas 14, 110704 (2007).

[14] S.H. Müller, A. Diallo, A. Fasoli, I. Furno, B. Labit, G. Plyushchev, M. Podestà, and F. M. Poli, Phys. Plasmas 13, 100701 (2006).

[15] S. L. Chen and T. Sekiguchi, J. Appl. Phys. 36, 2363 (1965).

[16] L. Giannone, R. Balbn, H. Niedermeyer, M. Endler, G. Herre, C. Hidalgo, A. Rudyj, G. Theimer, and Ph. Verplanke, Phys. Plasmas 1, 3614 (1994); V.I. Demidov, S.V. Ratynskaia, and K. Rypdal, Rev. Sci. Instrumen. 73, 3409 (2002).

[17] M. Podestà, A. Fasoli, B. Labit, I. Furno, P. Ricci, F.M. Poli, A. Diallo, S.H. Müller, and C. Theiler, Phys. Rev. Lett. 101, 045001 (2008).

[18] P.C. Stangeby, The plasma boundary of magnetic fusion devices, IOP publishing (2000).

[19] M. Podestà, Ph.D. thesis, Ecole Polythenique Federale de Lausanne, 2007, http://library.epfl.ch/en/theses/?nr=3765.

[20] M. Podestà, A. Fasoli, B. Labit, M. McGrath, S.H. Müller, and F.M. Poli, Plasma Phys. Control. Fusion 48, 1053 (2006).

[21] B. Labit, I. Furno, A. Fasoli, A. Diallo, S. H. Müller, G. Plyushchev, M. Podestà, and F. M. Poli, Phys. Rev. Lett. 98, 255002 (2007); B. Labit, A. Diallo, A. Fasoli et al., I. Furno, D. Iraji, S.H. Müller, G. Plyushchev, M Podestà, F.M. Poli, P. Ricci, C. Theiler, and J Horacek, Plasma Phys. Contrl. Fusion 49, B281 (2007).

[22] S.H. Müller, A. Fasoli, B. Labit, M. McGrath, O. Pisaturo, G. Plyushchev, M. Podestà, and F. M. Poli, Phys. Plasmas 12, 090906 (2005).

[23] C.Q. Yu, S. I. Krasheninnikov, and P. N. Guzdar, Phys. Plasmas 13, 042508 (2006).

[24] N. Bisai, A. Das, S. Deshpande, R. Jha, P. Kaw, A. Sen, and R. Singh, Phys. Plasmas 11, 4018 (2004). 
[25] V. Naulin, J. Nycander; J.J. Rasmussen, Phys. Rev. Lett. 81, 4148 (1998); O.E. Garcia, V. Naulin, A.H. Nielsen, and J. J. Rasmussen, Phys. Rev. Lett. 92, 165003 (2004).

[26] V. Naulin and A.H. Nielsen, Siam J. Sci. Comput. 25, 104 (2003). 
- Figure 1: (Color online) PDF (upper panels) and PSD (lower panels) for the $\tilde{I}_{\text {sat }}$ signals at $r=-4,4,12 \mathrm{~cm}$ (from left to right), evaluated from the simulation (solid black line) and HEXTIP (dashed red line).

- Figure 2: (Color online) Mean value, fluctuation, skewness, and kurtosis of the $I_{\text {sat }}$ signal, obtained through the HEXTIP array (red circles), SLP (green X marks), and the simulation results (solid black line).

- Figure 3: (Color online) Mean value, fluctuation, skewness, and kurtosis of the $V_{f l}$ signal, obtained through the HEXTIP array (red circles), SLP (green X marks), and the simulation results (solid black line).

- Figure 4: (Color online) Typical $I_{\text {sat }}$ values obtained from SLP (red dots) and from the virtual LP inserted in the simulations. The thin blue line interpolates the experimental values, the thick green line interpolates the simulation values.

- Figure 5: (Color online) Profile for $\bar{T}_{e}, \bar{n}$, and $\bar{\phi}$ obtained from the simulations (solid black line), SLP (red circles) and from the sweeping of the virtual LP in the simulation (black X marks).

- Figure 6: (Color online) Two points correlation technique results obtained for the simulations (left panels) and from SLP data (right panels) for $r=-4,0,4,8 \mathrm{~cm}$ (from top to bottom).

- Figure 7: (Color online) Results from the CA analysis from the simulation results (left) and the experimental data (right) for $\tau=-80,-12,8 \mu \mathrm{s}$ (from top to bottom).

- Figure 8: (Color online) Result from the structure analysis for the experiment (red dashed line) and the simulation (solid black line). The number of structure $N$, the vector of their averaged velocity $\mathbf{V}$, the average amplitude $H$, and the average area $A$, is shown as a function of $r$, for both positive (left) and negative structure (right). The velocity maximum modulus for positive structures is $1360 \mathrm{~m} / \mathrm{s}$ (experiment) and $2960 \mathrm{~m} / \mathrm{s}$ (simulation); for negative structures it is $2820 \mathrm{~m} / \mathrm{s}$ (experiment) and 2220 $\mathrm{m} / \mathrm{s}$ (simulation). 
- Figure 9: (Color online) Temperature fluctuations measured in the simulations (solid black line) and by the triple probe (dashed red line).

- Figure 10: (Color online) Transport evaluated using the structure analysis (upper panels) for positive (left) and negative (right) structures. The transport evaluated using the TP is shown in the lower panel. Solid black lines represent simulation data, red dashed lines the experimental measurements. 